\title{
SARS-Coronavirus-2 Nsp13 Possesses NTPase and RNA Helicase Activities That Can Be Inhibited by Bismuth Salts
}

\author{
Ting Shu ${ }^{1,2} \cdot$ Muhan Huang ${ }^{2} \cdot \mathrm{Di} \mathrm{Wu^{2 }} \cdot$ Yujie Ren ${ }^{2,3} \cdot$ Xueyi Zhang ${ }^{2} \cdot$ Yang Han $^{1,2} \cdot$ Jingfang $\mathrm{Mu}^{2} \cdot$ \\ Ruibing Wang ${ }^{4} \cdot$ Yang Qiu ${ }^{1,2,5} \cdot$ Ding-Yu Zhang $^{1}$ (i) $\cdot$ Xi Zhou $^{1,2,5}$ (i)
}

Received: 7 May 2020 / Accepted: 19 May 2020 / Published online: 4 June 2020

(C) Wuhan Institute of Virology, CAS 2020

\begin{abstract}
The ongoing outbreak of Coronavirus Disease 2019 (COVID-19) has become a global public health emergency. SARScoronavirus-2 (SARS-CoV-2), the causative pathogen of COVID-19, is a positive-sense single-stranded RNA virus belonging to the family Coronaviridae. For RNA viruses, virus-encoded RNA helicases have long been recognized to play pivotal roles during viral life cycles by facilitating the correct folding and replication of viral RNAs. Here, our studies show that SARS-CoV-2-encoded nonstructural protein 13 (nsp13) possesses the nucleoside triphosphate hydrolase (NTPase) and RNA helicase activities that can hydrolyze all types of NTPs and unwind RNA helices dependently of the presence of NTP, and further characterize the biochemical characteristics of these two enzymatic activities associated with SARS-CoV-2 nsp13. Moreover, we found that some bismuth salts could effectively inhibit both the NTPase and RNA helicase activities of SARS-CoV-2 nsp13 in a dose-dependent manner. Thus, our findings demonstrate the NTPase and helicase activities of SARS-CoV-2 nsp13, which may play an important role in SARS-CoV-2 replication and serve as a target for antivirals.
\end{abstract}

Keywords SARS-coronavirus-2 $($ SARS-CoV-2) $\cdot$ Nsp13 $\cdot$ NTPase $\cdot$ Helicase $\cdot$ Antiviral target

Ting Shu and Muhan Huang have contributed equally to this work.

Electronic supplementary material The online version of this article (https://doi.org/10.1007/s12250-020-00242-1) contains supplementary material, which is available to authorized users.

Ding-Yu Zhang

zhangdy63@hotmail.com

$\triangle$ Xi Zhou

zhouxi@wh.iov.cn

1 Center for Translational Medicine, Wuhan Jinyintan Hospital, Wuhan 430023, China

2 State Key Laboratory of Virology, Wuhan Institute of Virology, Center for Biosafety Mega-Science, Chinese Academy of Sciences, Wuhan 430071, China

3 Center for Precision Translational Medicine of Wuhan Institute of Virology and Guangzhou Women and Children's Medical Center, Guangzhou Women and Children's Medical Center, Guangzhou 510120, China

4 State Key Laboratory of Quality Research in Chinese Medicine, Institute of Chinese Medical Sciences, University of Macau, Macau SAR 999078, China

5 University of Chinese Academy of Sciences, Beijing 100049, China

\section{Introduction}

Coronaviruses (CoVs) are a large family of singlestranded, positive-sense RNA viruses that belong to the family Coronaviridae in the order Nidovirales (Cui et al. 2019). Coronavirus has a large RNA genome of $26-31 \mathrm{~kb}$ in length, and its replicase gene consists of two open reading frames (ORFs) 1a and $1 \mathrm{~b}$. These two ORFs yield two polyproteins, ppla and pplab, which are proteolytically cleaved into 16 nonstructural proteins (nsp1-16) that play pivotal roles in the life cycle of CoVs.

Coronaviruses can infect a variety of animals, including humans, bats, mice and birds (Zhou et al. 2020). Previously, six different coronaviruses had been found to infect humans, including CoV-229E, CoV-OC43, CoV-NL63, CoV-HKU1, SARS-CoV, and Middle East respiratory syndrome coronavirus (MERS-CoV) (Cui et al. 2019). Among them, CoV-229E, CoV-OC43, CoV-NL63, and CoV-HKU1 primarily cause mild and self-limiting diseases such as common cold, while SARS-CoV and MERS-CoV have caused severe respiratory illness.

The outbreak of a novel coronavirus, named SARSCoV-2, has emerged and rapidly spread to the globe since 
late December 2019 (Zhu et al. 2020), which has been declared as a pandemic by the World Health Organization (WHO). SARS-CoV-2 cause coronavirus disease 2019 (COVID-19), which manifests a wide spectrum of diseases, ranging from mild to severe symptoms such as pneumonia, acute respiratory distress syndrome, and even death (Chen et al. 2020; Guan et al. 2020; Huang et al. 2020; Wang et al. 2020; Yang et al. 2020a, b). The ongoing outbreak of SARS-CoV-2 has caused tremendous losses in human lives and economy around the world, while there is no approved drug or vaccine available for this virus.

For most RNA viruses including coronaviruses, viral RNAs (vRNAs) usually form a variety of cis-acting elements within the un-translational regions (UTRs) or ORF regions. These vRNA elements play indispensable roles in replication, translation and encapsidation, and need to be folded into correct secondary or tertiary structures to be functional. In addition, viral double-stranded RNAs (dsRNAs) produced during replication must be unwound to allow next round of vRNA replication. However, the proper folding of RNA elements or unwinding of dsRNAs is usually challenging, which requires the aid of RNA remodelling proteins including RNA helicase and RNA chaperones (Lorsch 2002; Bleichert and Baserga 2007; Musier-Forsyth 2010). Thus far, numerous RNA viruses have been found to encode their own RNA helicases, which are usually indispensable components of vRNA replication complexes (Yang et al. 2014; Xia et al. 2015; Jain et al. 2016; Li et al. 2018; Shu et al. 2019) and recognized as ideal targets for developing antivirals (Pfister and Wimmer 1999; Li et al. 2018; Shu et al. 2019).

Previous studies have shown that SARS-CoV nsp13 has an NTPase activity as well as an RNA helicase activity belonging to helicase superfamily-1 (Tanner et al. 2003; Ivanov et al. 2004). Considering the high homology of the amino acid sequences among coronaviral nsp13 proteins, it is intriguing to examine whether SARS-CoV-2 nsp13 also possesses the NTPase and RNA helicase activities. More importantly, we can re-examine the drugs that were found to target other coronavirus nsp13 for their effects on SARS-CoV-2 nsp13, which may accelerate the development of antivirals against COVID-19 in a timely manner. In this study, we expressed and purified recombinant nsp13 of SARS-CoV-2, and examined its biochemical activities. Our data show that SARS-CoV-2 nsp13 does possess the NTPase and RNA helicase activities that can hydrolyze all types of NTPs and unwind RNA helices in an NTPdependent manner, and some bismuth salts can effectively inhibit both the NTPase and RNA helicase activities of SARS-CoV-2 nsp13.

\section{Materials and Methods}

\section{Plasmid Construction}

Maltose-binding protein (MBP) fusion expression vector pMAL-SARS-CoV-2 nsp13 was generated using a standard cloning protocol. The cDNA fragment of SARS-CoV-2 nsp13 was generated by reverse transcription from the RNA template of SARS-CoV-2-infected cells. The SARSCoV-2 nsp13 genome fragment was inserted into the pMAL-c2X vector at $B a m \mathrm{H} \mathrm{I} / \mathrm{Sal}$ I restriction sites. The primers used in this study are shown in Supplementary Table S1.

\section{Expression and Purification of Recombinant Protein}

E. coli (BL21-DE3) cells were transformed by introduction of pMAL-SARS-COV-2 nsp13. In briefly, E. coli were grown at $37{ }^{\circ} \mathrm{C}$ and induced with $300 \mu \mathrm{mol} / \mathrm{L}$ IPTG when the OD value reached $\sim 0.6$. Thereafter, the induced E. coli were transferred to $22{ }^{\circ} \mathrm{C}$ to grow for $12 \mathrm{~h}$. Cells were harvested and lysed by ultra-sonication. After centrifugation at $12,000 \times g$, the proteins in the supernatant was purified using amylase affinity chromatography (New England BioLabs, Ipswich, MA) according to the manufacturer's protocol. All the purified proteins were concentrated using Amicon Ultra-30 filters (Millipore, Schwalbach, Germany). After that, the store buffer was exchanged to $50 \mathrm{mmol} / \mathrm{L}$ 2-[4-(2-hydroxyethyl)-1-piperazinyl] ethane sulfonic acid (HEPES)-KOH (pH 8.0). All proteins were quantified by the Bradford method and stored at $-80{ }^{\circ} \mathrm{C}$ in aliquots. Proteins were separated on $10 \%$ SDS-PAGE and visualized by Coomassie blue.

\section{NTPase Assay}

NTPase activities were performed by measuring the released inorganic phosphate during NTP hydrolysis via a direct colorimetric assay as previously described (Yang et al. 2017). The concentrations of inorganic phosphate were determined by matching the absorbance at wavelength $620 \mathrm{~nm}$ (A620) in a known standard inorganic phosphate curve. All of the data given by this quantitative assay were averages of three independently repeated experiments.

\section{Preparation of RNA Helix Substrate}

The RNA helix substrates consist of two complementary nucleic acid strands, one of which was labeled at $5^{\prime}$-end with Hexachloro-Fluorescein hosphoramidite (HEX) and 
the other was unlabeled. The labeled strands were purchased from TaKaRa (Dalian, China). All the unlabeled RNA strands were transcribed in vitro using T7 RNA polymerase (Promega, Madison, WI). The posttranscriptional RNAs were purified by using Poly-Gel RNA extraction kit (Omega Bio-Tek, Guangzhou, China) according to the manufacturer's instructions. The oligonucleotide helices were generated by annealing the labeled strand and unlabeled strand, at which mixed a 1:1 ratio in a $10-\mu \mathrm{L}$ reaction mixture containing $25 \mathrm{mmol} / \mathrm{L}$ HEPES$\mathrm{KOH}(\mathrm{pH} 8.0)$ and $25 \mathrm{mmol} / \mathrm{L} \mathrm{NaCl}$. The mixture was heated to $95^{\circ} \mathrm{C}$ for $5 \mathrm{~min}$ and was then cooled gradually to $25{ }^{\circ} \mathrm{C}$ to produce helical duplexes. Two RNA helix substrate with both $5^{\prime}$ - and $3^{\prime}$-protrusions was annealed with RNA1 and RNA2 (24-nt non-labeled) or RNA3 (28-nt nonlabeled). The $5^{\prime}$-protruded RNA helix was annealed with RNA1 and RNA4. The 3'-protruded RNA helix was annealed with RNA1 and RNA5. The blunt RNA helix was annealed with RNA1 and RNA6. All the oligonucleotides are listed in Supplementary Table S2.

\section{RNA Helix Unwinding Assay}

The standard helix destabilizing assay was performed as previously described (Shu et al. 2019). Briefly, the indicated recombinant protein and $0.1 \mathrm{pmol} / \mathrm{L}$ of HEX-labeled helix substrate were added to the standard reaction mix containing $50 \mathrm{mmol} / \mathrm{L}$ HEPES-KOH (pH 8.0), $50 \mathrm{mmol} / \mathrm{L}$ $\mathrm{NaCl}, 2 \mathrm{mmol} / \mathrm{L} \mathrm{MgCl}_{2}, 5 \mathrm{mmol} / \mathrm{L}$ ATP and $20 \mathrm{U}$ RNasin (Promega). After incubation at $37^{\circ} \mathrm{C}$, the reaction was terminated by adding $10 \times$ loading buffer $[100 \mathrm{mmol} / \mathrm{L}$ Tris- $\mathrm{HCl}, 1 \%$ SDS, 50\% glycerol, and bromophenol blue ( $\mathrm{pH}$ 7.5)]. The mixtures were then electrophoresed on $15 \%$ native-PAGE gels, followed by scanning with a Typhoon 9500 imager (GE Healthcare, Piscataway, NJ).

\section{Bismuth Salts}

Bismuth potassium citrate (CAS No. 57644-54-9) was purchased from MedChemExpress. Ranitidine bismuth citrate (CAS No. 128345-62-0) was purchased from Changzhou Lanling Pharmaceutical Co., Ltd. Bismuth citrate (CAS No. 813-93-4) was purchased from SigmaAldrich. These compounds were serially diluted to the indicated concentrations and added to the ATPase and helix unwinding reactions, respectively.

\section{Results}

\section{SARS-CoV-2 Nsp13 Contains NTPase Activity}

Previous studies have reported SARS-CoV nsp13 contains NTPase and helicase activities (Tanner et al. 2003; Ivanov et al. 2004). To characterize the biochemical activities of SARS-CoV-2 nsp13, we expressed and purified it as a maltose-binding protein (MBP-nsp13) from E. coli prokaryotic expression system (Supplementary Fig. S1).

The RNA-helix unwinding of helicases usually requires NTP binding and hydrolysis to provide energy. Therefore, we first sought to examine whether SARS-CoV-2 nsp13 has the NTPase activity to hydrolyze four kinds of NTPs by measuring the released inorganic phosphate via a sensitive colorimetric assay. We found that the recombinant SARSCoV-2 nsp13 could hydrolyze all four types of NTPs, with a preference for ATP and GTP (Fig. 1A). We used ATP in the subsequent assays, as it is the major energy source in cells. Further investigation showed that the amount of hydrolysed ATP by nsp13 was increased with the increasing concentrations of nsp13 used in the reaction mix (Fig. 1B). Moreover, we found that the NTPase activity of SARS-CoV-2 nsp13 requires the presence of divalent metallic ions, as our data showed that $2 \mathrm{mmol} / \mathrm{L} \mathrm{Mg}^{2+}$, $\mathrm{Mn}^{2+}, \mathrm{Ca}^{2+}$, or $\mathrm{Zn}^{2+}$ could support the ATPase activity of nsp13, and their efficiencies were as follow: $\mathrm{Mg}^{2+}>-$ $\mathrm{Mn}^{2+}>\mathrm{Zn}^{2+}>\mathrm{Ca}^{2+}$ (Fig. 1C). Besides, SARS-CoV-2 nsp13 displays its optimal ATPase activity in the presence of $2 \mathrm{mmol} / \mathrm{L} \mathrm{Mg}^{2+}$, while higher concentrations of $\mathrm{Mg}^{2+}$ showed certain inhibitory effect on the ATPase activity (Fig. 1D). Together, our data show that SARS-CoV-2 nsp13 possesses an NTPase activity, which is dependent on the presence of certain divalent metallic ions.

\section{SARS-CoV-2 Nsp13 Has the RNA Helix Unwinding Activity}

After establishing that SARS-CoV-2 nsp13 possesses the activity to hydrolyze NTP, we sought to examine whether it has the RNA helix unwinding activity. To this end, we constructed an RNA helix substrate with both $5^{\prime}$ - and $3^{\prime}$ single-stranded protrusions by annealing a 24-nt nonlabeled RNA with a 42-nt HEX-labeled RNA (as illustrated in Fig. 2A). This RNA helix substrate was commonly used to characterize the helix unwinding activity of RNA helicases (Li et al. 2018; Shu et al. 2019). The helix unwinding assay was performed by incubating the RNA helix substrate with MBP-nsp13 in a standard unwinding reaction mix containing ATP and $\mathrm{MgCl}_{2}$, followed by the separation of the RNA substrate strands via electrophoresis. As shown in Fig. 2B, HEX-labeled RNA strand was efficiently 
A

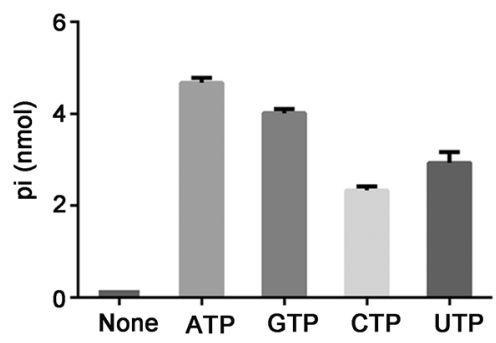

C

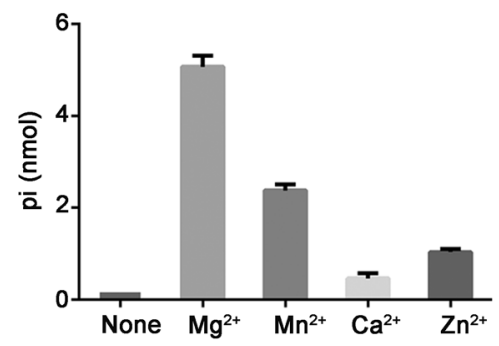

B

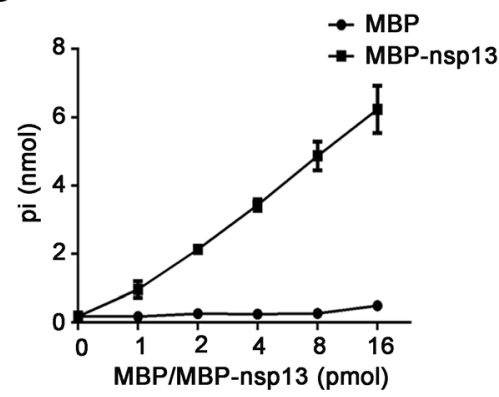

D

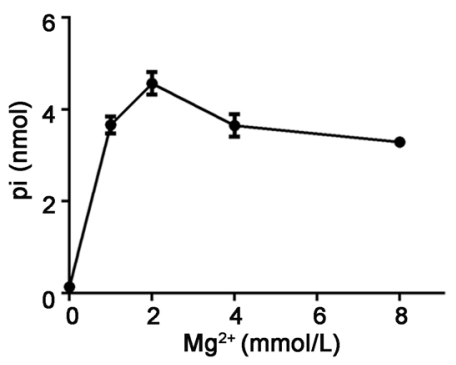

with $2.5 \mathrm{mmol} / \mathrm{L}$ ATP at $2 \mathrm{mmol} / \mathrm{L}$ indicated divalent metal ions. The reaction without any divalent metal ion was used as negative control (None). D $10 \mathrm{pmol} / \mathrm{L} \mathrm{MBP-nsp13}$ was reacted with $2.5 \mathrm{mmol} / \mathrm{L}$ ATP at the indicated concentrations of $\mathrm{MgCl}_{2}$. $\mathrm{MBP}$ alone was used as the negative control. Error bars represent standard deviation (SD) values from three separate experiments.
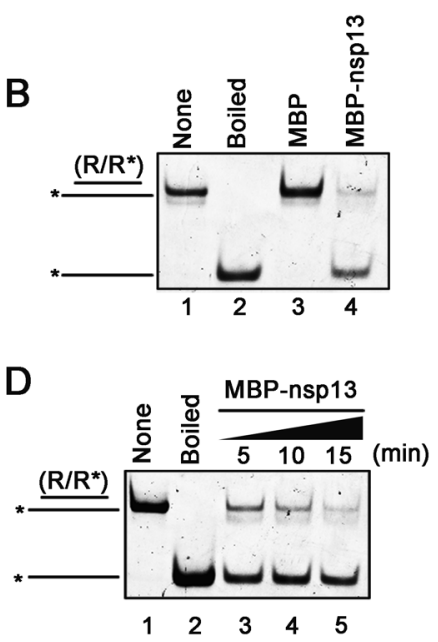

(lane 1) and reaction mixture with MBP alone (lane 3) were used as negative controls, and boiled reaction mixture (lane 2) was used as positive control. C The RNA helix unwinding assay was performed in the presence of increasing concentrations of MBP-nsp13. D MBPnsp13 (20 pmol/L) was reacted with the RNA helix substrate $(0.1 \mathrm{pmol} / \mathrm{L})$ in reaction mixture at different reaction time.

substrates were used as the positive control (lane 2). Moreover, when increasing concentrations of MBP-nsp13 were incubated with the RNA helix substrate, MBP-nsp13 can efficiently unwind RNA helix in a dose-dependent 
manner (Fig. 2C). Besides, our data showed that the released HEX-labeled RNAs were gradually increased along with the increasing reaction time (Fig. 2D).

We further characterized the helix unwinding activity by MBP-nsp13 via incubating it with the RNA helix substrate (Fig. 3A) under different conditions. We found that MBPnsp13 could only efficiently unwind the the RNA helix substrate in the presence of ATP and GTP, whereas the efficiency of RNA helix-unwinding by nsp13 was limited in the presence of CTP and UTP (Fig. 3B), consistent with the NTP preference of the NTPase activity of nsp13. Besides, our data showed that the RNA helix-unwinding by MBP-nsp13 required the presence of divalent metallic ions. This protein showed optimal helix unwinding activity in the presence of $\mathrm{Mg}^{2+}$ (Fig. 3C, lane 4), while the presence of $\mathrm{Mn}^{2+}$ also supported nsp13 to unwind RNA duplex in a lesser extent than that of $\mathrm{Mg}^{2+}$ (Fig. 3C, lane 5). On the contrast, the presence of $\mathrm{Ca}^{2+}$ or $\mathrm{Zn}^{2+}$ failed to support the helix unwinding activity of SARS-CoV-2 nsp13 (Fig. 3C, lanes 6 and 7). And this result is consistent with the observation that the nsp13 NTPase activity is only minimal in the presence of $\mathrm{Ca}^{2+}$ or $\mathrm{Zn}^{2+}$ (Fig. 1C). Moreover, SARS-CoV-2 nsp13 displayed the optimal helicase activity in the presence of $2 \mathrm{mmol} / \mathrm{L} \mathrm{Mg}^{2+}$, while higher concentrations of $\mathrm{Mg}^{2+}$ even showed inhibitory effect (Fig. 3D), consistent with the results from Fig. 1D. Interestingly, high concentration of multivalent metallic cations, such as $\mathrm{Mg}^{2+}$ or $\mathrm{Zn}^{2+}$, exhibited inhibitory effects on multiple viral RNA helicases, which is probably due to stabilizing RNA conformation or competing with RNA helicases for nonspecific RNA binding.

Taken together, our findings showed that SARS-CoV-2 nsp13 has the RNA helicase activity in the presence of ATP and $\mathrm{Mg}^{2+}$.

A
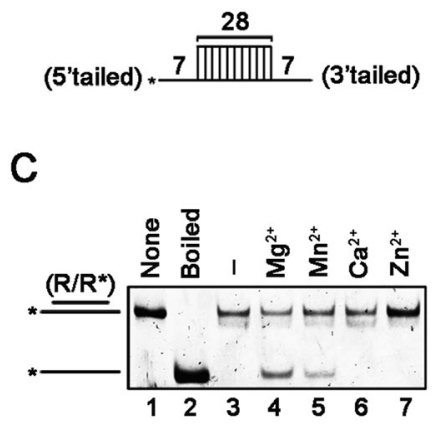

Fig. 3 Optimal biochemical reaction conditions for the RNA helix unwinding activity of SARS-CoV-2 nsp13. A Schematic illustration of the RNA helix substrate $\left(\mathrm{R} / \mathrm{R}^{*}\right)$. Asterisks indicate the HEXlabelled strands. B-D MBP-nsp13 (20 pmol/L) was reacted with the
SARS-CoV-2 Nsp13 Unwinds RNA Helix in the 5'3' Directionality

The directionality of helix-unwinding is one of the fundamental characteristics of RNA helicases (Musier-Forsyth 2010). After establishing that SARS-CoV-2 nsp13 displays the RNA helix-unwinding activity, we sought to characterize its helix unwinding directionality. To this end, we constructed three different RNA helix substrates with $5^{\prime}$ protruded, 3'-protruded and blunted ends, respectively (Fig. 4A-Fig. 4C). Then, we reacted these helix substrates with the purified MBP-nsp13 in the standard helixunwinding assay. Our data showed that SARS-CoV-2 nsp13 efficiently unwound the RNA helix substrate with $5^{\prime}$-protrusion (Fig. 4D, lane 3), but not the substrate with 3 '-protrusion (Fig. 4D, lane 6). Moreover, the blunt ended helix substrate could not be unwounded by MBP-nsp13 (Fig. 4E). Together, our findings indicated that SARSCoV-2 nsp13 is able to unwind RNA helix in the directionality of $5^{\prime}-3^{\prime}$, which is consistent with other coronaviral RNA helicases.

\section{Bismuth Salts Inhibit the NTPase and RNA Helix- Unwinding Activities of SARS-CoV-2 Nsp13}

As aforementioned, we have observed that certain divalent cations, like $\mathrm{Ca}^{2+}$ and $\mathrm{Zn}^{2+}$, did not support RNA helix unwinding by SARS-CoV-2 nsp13, while high concentrations of $\mathrm{Mg}^{2+}$ showed inhibitory effect (Fig. 3C, 3D). Moreover, previous studies reported that some bismuth salts could inhibit the NTPase and RNA helicase activities of SARS-CoV nsp13 as well as the replication of SARS$\mathrm{CoV}$ in cells (Yang et al. 2007a, b). Therefore, we sought to examine whether NTPase and RNA helix-unwinding activities of SARS-CoV-2 nsp13 can also be inhibited by
B

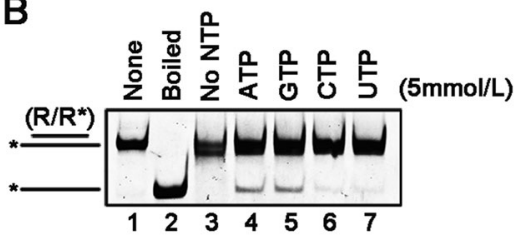

D

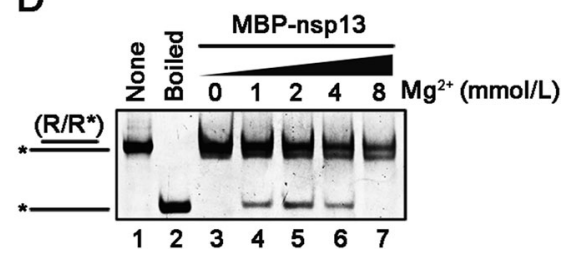

RNA helix substrate $(0.1 \mathrm{pmol} / \mathrm{L})$ in the presence of the indicated NTPs $(5 \mathrm{mmol} / \mathrm{L})($ B $)$ each indicated divalent metal ion $(2 \mathrm{mmol} / \mathrm{L}$ for each) (C), increasing concentrations of $\mathrm{MgCl}_{2}$ (D). 

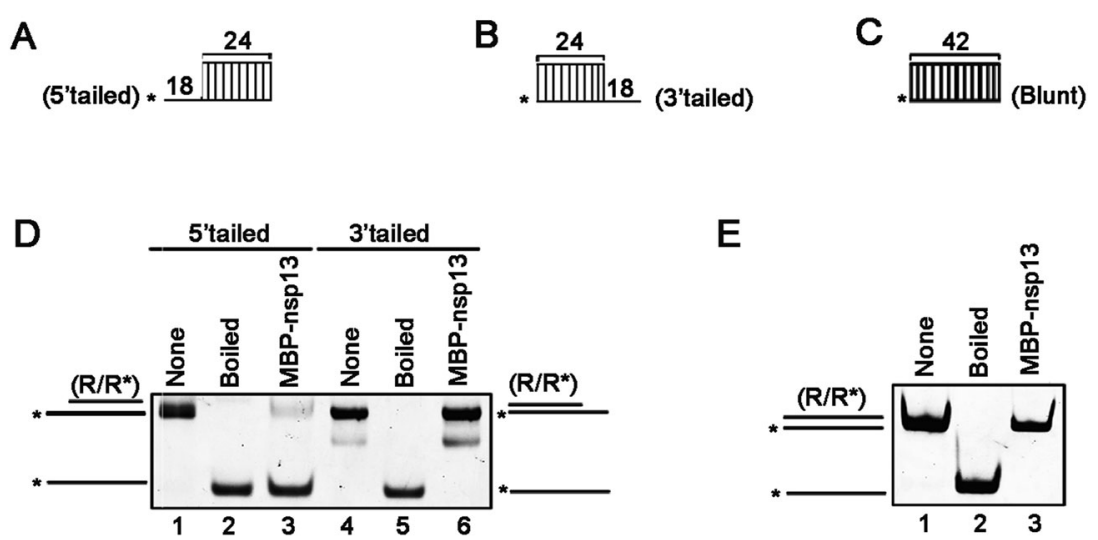

Fig. 4 SARS-CoV-2 nsp13 unwinds RNA helix in the $5^{\prime}-3^{\prime}$ directionality. A-C Schematic illustrations of the RNA helix substrates with $5^{\prime}$-tailed (A), $3^{\prime}$-tailed (B), and blunt ends $(\mathbf{C})$. Asterisks indicate the HEX-labelled strand. D MBP-nsp13 (20 pmol/L) was reacted with

the bismuth salts. Here, we used three different bismuth salts, including bismuth potassium citrate (BPC), ranitidine bismuth citrate (RBC), and bismuth citrate (BC). BPC and $\mathrm{RBC}$ are originally used to treat the gastrointestinal diseases, and BC is the intermediate of BPC. Our data showed that all these bismuth salts inhibited the ATPase activity of SARS-CoV-2 nsp13 at $10 \mu \mathrm{mol} / \mathrm{L}$, as BPC and RBC showed stronger inhibitory effects than BC (Fig. 5A). Moreover, we found that $10 \mu \mathrm{mol} / \mathrm{L} \mathrm{BPC}$ or RBC almost abolished the RNA helix unwinding activity of nsp13 (Fig. 5B, lanes 4 and 5); on the other hand, although $\mathrm{BC}$ treatment also effectively inhibited the RNA helicase activity of nsp13, its inhibitory efficiency was in much less than BPC or RBC (Fig. 5B, lane 6). To further characterize the inhibitory effect of BPC or RBC on the NTPase and RNA helicase activities of SARS-CoV-2, we treated recombinant nsp13 with increasing concentrations of either bismuth salts. Our data show that either BPC or RBC could inhibit the ATPase and helicase activities of MBP-nsp13 in a dose-dependent manner (Fig. 6).

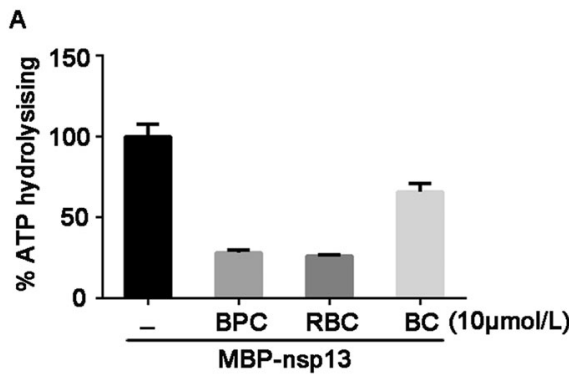

Fig. 5 Bismuth salts inhibit the ATPase and RNA helix unwinding activities of SARS-CoV-2 nsp13. A The ATPase activity of MBPnsp13 $(10 \mathrm{pmol} / \mathrm{L})$ in the presence of the indicated bismuth salts (10 $\mu \mathrm{mol} / \mathrm{L}$ for each). $\mathrm{X}$-axis were expressed as the different bismuth salts. Values (Y-axis) were expressed as percentages of those of mock-treated (-) ATPase activity of MBP-nsp13 (10 pmol/L). The
$0.1 \mathrm{pmol} / \mathrm{L} 5^{\prime}$-tailed (lane 3 ) or $3^{\prime}$-tailed (lane 6) RNA helix substrate. E MBP-nsp13 (20 pmol/L) was reacted with $0.1 \mathrm{pmol} / \mathrm{L}$ helix substrate with blunt ends.

In conclusion, our data show that the bismuth salt, BPC or RBC, can effectively inhibit both the NTPase and RNA helicase activities of SARS-CoV-2 nsp13 dosedependently.

\section{Discussion}

The ongoing COVID-19 outbreak in China and across the globe has become a significant threat to the health and lives of humans. Given the emergency and threat caused by the SARS-CoV-2 epidemic, better understanding all aspects of SARS-CoV-2 virology and uncovering potential antiviral drug targets are urgently needed. Virus-encoded RNA helicases are a class of viral enzymes that are parts of viral RNA replication complex and play indispensable roles in viral propagation and pathogenesis. Therefore, virusencoded RNA helicases are also recognized as ideal targets for antiviral drugs. In this study, we found that SARS-CoV-2 nsp13 possesses the NTPase and RNA helicase activities

B
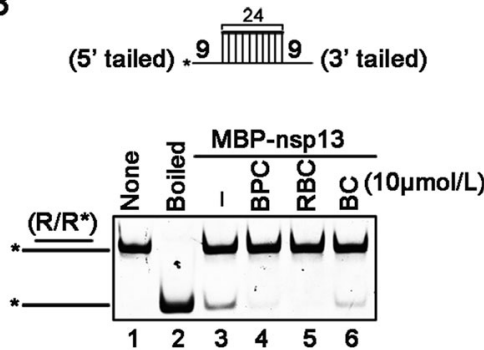

error bars represent SD values from three separate experiments. B Upper panel: schematic illustration of the RNA helix substrate (R/R*); asterisks indicate the HEX-labelled strand. Lower panel: The RNA helix unwinding assays were performed by incubating $0.1 \mathrm{pmol} / \mathrm{L}$ RNA helix substrate with MBP-nsp13 (2 pmol/L) in presence of the indicated bismuth salts $(10 \mu \mathrm{mol} / \mathrm{L}$ for each $)$. 
Fig. $6 \mathrm{BPC}$ and $\mathrm{RBC}$ inhibit the ATPase and RNA helix unwinding activities of SARSCoV-2 nsp13 in a dosedependent manner. A and $\mathbf{D}$ The ATPase activity of MBP-nsp13 $(10 \mathrm{pmol} / \mathrm{L})$ was performed in the presence of increasing concentrations of BPC $(\mathbf{A})$ or RBC (D) as indicated. B and E The RNA helix unwinding activity of MBP-nsp13 (2 pmol/ L) was performed in the presence of increasing concentrations of BPC (B) or RBC (E) as indicated. $\mathbf{C}$ and F The unwinding activities in (B and $\mathbf{E}$ ) were plotted as percentages of the released HEX-labelled RNA from the total RNA helix (Y-axis) at indicated concentrations (Xaxis) of BPC (C) or RBC (F). The error bars represent SD values from three separate experiments.
A

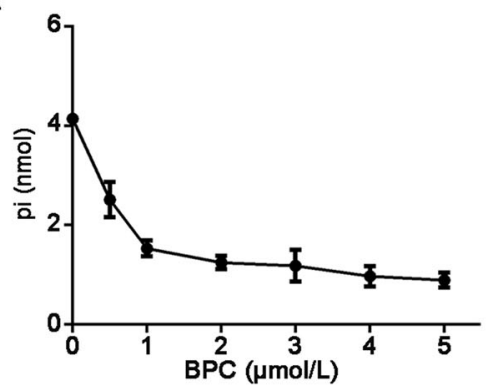

B

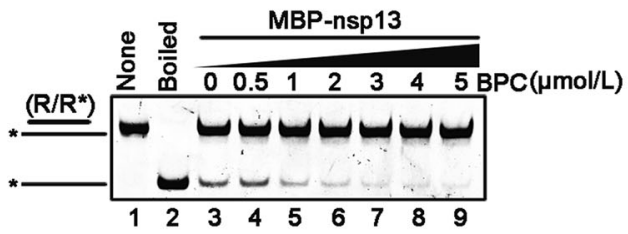

C

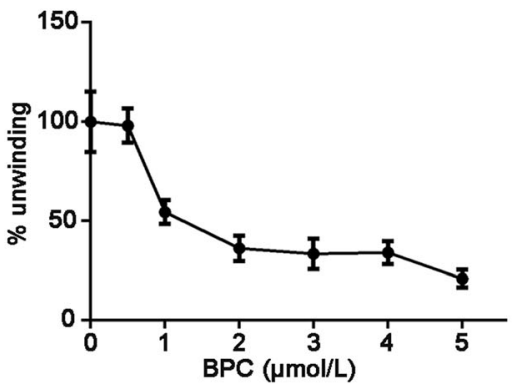

D

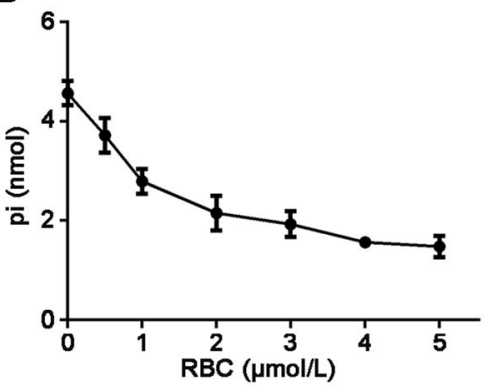

E

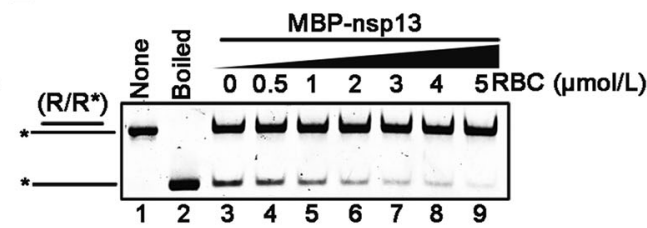

$\mathbf{F}$

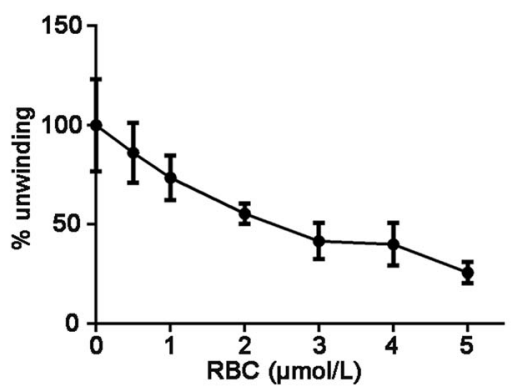

that can hydrolyze all types of NTPs and unwind RNA helix in an ATP-dependent manner. Moreover, our data showed that two bismuth salts, BPC and RBC, which are used in clinical treatment of gastrointestinal diseases, can effectively inhibit the NTPase and helicase activities of SARS-CoV-2 nsp13. From this perspective, our findings provide insights into the key viral replicative enzyme that probably represents an attractive target for developing antivirals.

The finding that SARS-CoV-2 nsp13 is able to hydrolyze NTPs and to destabilize RNA helix consistent with the previous observation that other coronaviral nsp13 proteins also possess the NTPase and RNA helicase activities (Tanner et al. 2003; Ivanov et al. 2004), implying the conserved and indispensable role of nsp13 in the life cycles of coronaviruses. Indeed, coronavirus nsp13 is a multifunctional protein with a Zinc-binding domain (ZBD) in $\mathrm{N}$-terminus and a helicase domain with typical conserved motifs of superfamily-1 (SF1) helicases are present in the C-terminal half (Subissi et al. 2014). The N-terminus of nsp13 are highly conserved and predicted to form a $\mathrm{Zn}^{2+}$ binding cluster, which conserved in all coronaviruses and nidoviruses (Seybert et al. 2005).

RNA helicases contain NTPase activity and utilize the energy of hydrolyzing ATP to melt base pairings, and are generally believed to play important roles in all processes involving RNAs during viral life cycles, including replication, transcription, translation as well as encapsidation (Bleichert and Baserga 2007; Musier-Forsyth 2010). For example, during the process of vRNA replication of RNA viruses, viral dsRNA replicative intermediates must be efficiently unwound to release nascently synthesized progeny vRNAs from template vRNAs (Yang et al. 2014; Xia et al. 2015). Therefore, virus-encoded RNA helicases have long been considered as the potential targets for antiviral therapy development. A serial of potential drugs, including flavonoids, myricetin, scultellarein, dihydroxychromones, aryl diketoacids, 3-[(2-nitrophenyl)sulphanylmethyl]4prop-2-enyl-1H-1,2,4-triazole-5-thione and bismuth salts had been found by different groups to target the ATPase/ helicase activities of SARS-CoV nsp13 (Seybert et al. 2005; Yang et al. 2007a, b; Lee et al. 2009; Adedeji et al. 2012; Yu et al. 2012). Similarly, we also found that bismuth salts BPC and RBC could effectively inhibit the ATPase and helicase activities of SARS-CoV-2 nsp13 in a dose-dependent manner. Because bismuth cation $\left(\mathrm{Bi}^{3+}\right)$ can bind strongly to metallothionein through the formation of Bi-S bonds (Sun et al. 1999), it is possible that BPC or $\mathrm{RBC}$ inhibits the enzymatic activities of SARS-CoV-2 nsp13 via binding to the N-terminal ZBD of nsp13. 
One of the symptoms of COVID-19 is diarrhoea and gastrointestinal disorder (Chen et al. 2020; Guan et al. 2020; Wang et al. 2020). Besides, SARS-CoV-2 could be found in the rectal swabs and feces from many COVID-19 patients. More importantly, the rectal swabs can detect SARS-CoV-2 in patients even when the testes for throat swabs turned negative, implying that SARS-CoV-2 is more persistent in gastrointestinal tract compared to that in respiratory tract. These findings imply that the gastrointestinal tract is also the target of SARS-CoV-2 and the risk of further dissemination of this virus to healthy population through fecal-oral route cannot be omitted. Since BPC and $\mathrm{RBC}$ are already used to treat disorders in gastrointestinal tract, they may have the potential to be further developed for the prevention and treatment of COVID-19. Future study should evaluate the inhibitory effect of BPC or RBC on SARS-CoV-2 replication in cells and in vivo.

In summary, SARS-CoV-2 nsp13 possesses the NTPase and RNA helicase activities that can hydrolyze all types of NTPs and unwind RNA helices dependently of the presence of NTP. Moreover, bismuth salts, such as BPC and $\mathrm{RBC}$, have been found to inhibit both the NTPase and RNA helicase activities of SARS-CoV-2 nsp13 in a dose-dependent manner. In a timely manner, our study extends our knowledge about one of the key replicative enzymes of SARS-CoV-2 and suggests that SARS-CoV-2 nsp13 can be a valuable target for antivirals, which may be helpful in the efforts to control this life-threatening virus.

Acknowledgements We wish to thank Prof. P. Zhou (Wuhan, China) for kindly providing materials. This work was supported by the Strategic Priority Research Program of CAS (XDB29010300 to X.Z.), National Natural Science Foundation of China (81873964 to Y.Q., 31800140 to J.M. and 31670161 to X.Z.), National Science and Technology Major Project (2018ZX10101004 to X.Z.), and the Science and Technology Development Fund, Macau SAR (0007/2020/A to R.W).

Author Contributions TS, MH, DW, YR, X Zhang, YH, JM and RW performed experiments. TS, YQ, DZ, and X Zhou designed the experiments and interpreted the results. YQ, DZ and $\mathrm{X}$ Zhou wrote and finalized the manuscript.

\section{Compliance with Ethical Standards}

Conflict of interest The authors declare that they have no conflicts of interest.

Animal and Human Rights Statement This article does not contain any studies with human or animal subjects performed by any of the authors.

\section{References}

Adedeji AO, Singh K, Calcaterra NE, DeDiego ML, Enjuanes L, Weiss S, Sarafianos SG (2012) Severe acute respiratory syndrome coronavirus replication inhibitor that interferes with the nucleic acid unwinding of the viral helicase. Antimicrob Agents Chemother 56:4718-4728

Bleichert F, Baserga SJ (2007) The long unwinding road of RNA helicases. Mol Cell 27:339-352

Chen N, Zhou M, Dong X, Qu J, Gong F, Han Y, Qiu Y, Wang J, Liu Y, Wei Y, Xia J, Yu T, Zhang X, Zhang L (2020) Epidemiological and clinical characteristics of 99 cases of 2019 novel coronavirus pneumonia in Wuhan, China: a descriptive study. Lancet S0140-6736(20):30211-30217

Cui J, Li F, Shi ZL (2019) Origin and evolution of pathogenic coronaviruses. Nat Rev Microbiol 17:181-192

Guan WJ, Ni ZY, Hu Y, Liang WH, Ou CQ, He JX, Liu L, Shan H, Lei CL, Hui DSC, Du B, Li LJ, Zeng G, Yuen KY, Chen RC, Tang CL, Wang T, Chen PY, Xiang J, Li SY, Wang JL, Liang ZJ, Peng YX, Wei L, Liu Y, Hu YH, Peng P, Wang JM, Liu JY, Chen Z, Li G, Zheng ZJ, Qiu SQ, Luo J, Ye CJ, Zhu SY, Zhong NS (2020) Clinical characteristics of coronavirus disease 2019 in China. N Engl J Med 382:1708-1720

Huang C, Wang Y, Li X, Ren L, Zhao J, Hu Y, Zhang L, Fan G, Xu J, Gu X, Cheng Z, Yu T, Xia J, Wei Y, Wu W, Xie X, Yin W, Li H, Liu M, Xiao Y, Gao H, Guo L, Xie J, Wang G, Jiang R, Gao Z, Jin Q, Wang J, Cao B (2020) Clinical features of patients infected with 2019 novel coronavirus in Wuhan, China. Lancet S0140-6736(20):30183-30185

Ivanov KA, Thiel V, Dobbe JC, van der Meer Y, Snijder EJ, Ziebuhr J (2004) Multiple enzymatic activities associated with severe acute respiratory syndrome coronavirus helicase. J Virol 78:5619-5632

Jain R, Coloma J, Garcia-Sastre A, Aggarwal AK (2016) Structure of the NS3 helicase from Zika virus. Nat Struct Mol Biol 23:752-754

Lee C, Lee JM, Lee NR, Kim DE, Jeong YJ, Chong Y (2009) Investigation of the pharmacophore space of Severe Acute Respiratory Syndrome coronavirus (SARS-CoV) NTPase/helicase by dihydroxychromone derivatives. Bioorg Med Chem Lett 19:4538-4541

Li TF, Hosmillo M, Schwanke H, Shu T, Wang Z, Yin L, Curry S, Goodfellow IG, Zhou X (2018) Human norovirus NS3 Has RNA helicase and chaperoning activities. J Virol 92:e01606-e01617

Lorsch JR (2002) RNA chaperones exist and DEAD box proteins get a life. Cell 109:797-800

Musier-Forsyth K (2010) RNA remodeling by chaperones and helicases. RNA Biol 7:632-633

Pfister T, Wimmer E (1999) Characterization of the nucleoside triphosphatase activity of poliovirus protein $2 \mathrm{C}$ reveals a mechanism by which guanidine inhibits poliovirus replication. J Biol Chem 274:6992-7001

Seybert A, Posthuma CC, van Dinten LC, Snijder EJ, Gorbalenya AE, Ziebuhr J (2005) A complex zinc finger controls the enzymatic activities of nidovirus helicases. J Virol 79:696-704

Shu T, Gan T, Bai P, Wang X, Qian Q, Zhou H, Cheng Q, Qiu Y, Yin L, Zhong J, Zhou X (2019) Ebola virus VP35 has novel NTPase and helicase-like activities. Nucl Acids Res 47:5837-5851

Subissi L, Imbert I, Ferron F, Collet A, Coutard B, Decroly E, Canard B (2014) SARS-CoV ORF1b-encoded nonstructural proteins 12-16: replicative enzymes as antiviral targets. Antiviral Res 101:122-130

Sun H, Li H, Harvey I, Sadler PJ (1999) Interactions of bismuth complexes with metallothionein(II). J Biol Chem 274:29094-29101

Tanner JA, Watt RM, Chai YB, Lu LY, Lin MC, Peiris JS, Poon LL, Kung HF, Huang JD (2003) The severe acute respiratory syndrome (SARS) coronavirus NTPase/helicase belongs to a distinct class of 5' to 3 ' viral helicases. J Biol Chem 278:39578-39582 
Wang D, Hu B, Hu C, Zhu F, Liu X, Zhang J, Wang B, Xiang H, Cheng Z, Xiong Y, Zhao Y (2020) Clinical characteristics of 138 hospitalized patients with 2019 novel coronavirus-infected pneumonia in Wuhan, China. JAMA 323(11):1061-1069

Xia H, Wang P, Wang GC, Yang J, Sun X, Wu W, Qiu Y, Shu T, Zhao X, Yin L, Qin CF, Hu Y, Zhou X (2015) Human enterovirus nonstructural protein 2CATPase functions as both an RNA helicase and ATP-independent RNA chaperone. PLoS Pathog 11:e1005067

Yang N, Tanner JA, Wang Z, Huang JD, Zheng BJ, Zhu N, Sun H (2007a) Inhibition of SARS coronavirus helicase by bismuth complexes. Chem Commun (Camb) 14:4413-4415

Yang N, Tanner JA, Zheng BJ, Watt RM, He ML, Lu LY, Jiang JQ, Shum KT, Lin YP, Wong KL, Lin MC, Kung HF, Sun H, Huang JD (2007b) Bismuth complexes inhibit the SARS coronavirus. Angew Chem Int Ed Engl 46:6464-6468

Yang J, Cheng Z, Zhang S, Xiong W, Xia H, Qiu Y, Wang Z, Wu F, Qin CF, Yin L, Hu Y, Zhou X (2014) A cypovirus VP5 displays the RNA chaperone-like activity that destabilizes RNA helices and accelerates strand annealing. Nucl Acids Res 42:2538-2554

Yang J, Qian Q, Li TF, Yang X, Won SJ, Zhou X (2017) Cypovirus capsid protein VP5 has nucleoside triphosphatase activity. Virol Sin 32:328-330
Yang X, Yang Q, Wang Y, Wu Y, Xu J, Yu Y, Shang Y (2020a) Thrombocytopenia and its association with mortality in patients with COVID-19. J Thromb Haemost. https://doi.org/10.1111/jth. 14848

Yang X, Yu Y, Xu J, Shu H, Xia J, Liu H, Wu Y, Zhang L, Yu Z, Fang M, Yu T, Wang Y, Pan S, Zou X, Yuan S, Shang Y (2020b) Clinical course and outcomes of critically ill patients with SARS-CoV-2 pneumonia in Wuhan, China: a single-centered, retrospective, observational study. Lancet Respir Med S2213-2600(20):30079-5

Yu MS, Lee J, Lee JM, Kim Y, Chin YW, Jee JG, Keum YS, Jeong YJ (2012) Identification of myricetin and scutellarein as novel chemical inhibitors of the SARS coronavirus helicase, nsP13. Bioorg Med Chem Lett 22:4049-4054

Zhou P, Yang XL, Wang XG, Hu B, Zhang L, Zhang W, Si HR, Zhu Y, Li B, Huang CL, Chen HD, Chen J, Luo Y, Guo H, Jiang RD, Liu MQ, Chen Y, Shen XR, Wang X, Zheng XS, Zhao K, Chen QJ, Deng F, Liu LL, Yan B, Zhan FX, Wang YY, Xiao GF, Shi ZL (2020) A pneumonia outbreak associated with a new coronavirus of probable bat origin. Nature 579:270-273

Zhu N, Zhang D, Wang W, Li X, Yang B, Song J, Zhao X, Huang B, Shi W, Lu R, Niu P, Zhan F, Ma X, Wang D, Xu W, Wu G, Gao GF, Tan W (2020) A novel coronavirus from patients with pneumonia in China, 2019. N Engl J Med 382:727-733 